\title{
Congress of the Spanish Society of Medical Oncology (SEOM) in Barcelona
}

\author{
Ramon Colomer
}

$\mathrm{T}$ he Spanish Society of Medical Oncology held its twelfth national congress on 21-23 October 2009.

In Spain, the specialty of Medical Oncology started almost 30 years ago, and essentially all medical oncologists and oncologists in training are members of SEOM, which now has more than 1200 members. The majority of medicines for cancer therapies are administered and investigated in Spain by medical oncologists, a situation that is slightly different to that in other countries in Europe, where several specialties may be implicated. This makes SEOM a very participative society, which is becoming increasingly influential in Spanish public opinion.

The SEOM meeting in 2009 was an outstanding success. There was a $29 \%$ increase in the number of attendees with respect to the last meeting, reaching more than 1100 participants. This is quite remarkable in the context of a global crisis and indicates Spanish oncologists' interest in the meeting. More than 450 abstracts were submitted this year and there were 150 speakers. Some of the innovations of the 2009 meeting included specific training sessions for residents and fellows, and sessions dedicated to clinical trial organisation and data management.

Two of the opening session lectures dealt with history. First, the new book History of Medical Oncology in
Spain was presented and, after that, the role of the former SEOM president Pau Viladiu in breast cancer research was reviewed. The Presidential Session had three presentations by outstanding Spanish Oncologists that showed the audience the extraordinary level and worldwide influence of Spanish research. This year, the SEOM meeting had two Multidisciplinary Symposia, one about tumours of the CNS and one about colorectal cancer liver metastases. Specialists from Radiation Therapy, Pathology, Imaging, Surgery and Neurology participated in these sessions.

During the meeting, SEOM awarded prizes for clinical research in cancer and other categories such as prizes for information about cancer, with a total of $€ 400,000$.

A novel experience in this meeting was the organisation of a session in which 14 of the Spanish cooperative groups of cancer research showed their achievements and plans for the future. The number of cancer patients that have participated in one of the studies of the Spanish cooperative groups is approaching 100,000, which is an outstanding achievement.

In conclusion, the 2009 meeting of the Spanish Society of Medical Oncology showed the vitality of the specialty and the progress of the SEOM. The future of Medical Oncology in Spain looks bright and encouraging.

R. Colomer (西)

President, Spanish Society of Medical Oncology (SEOM)

C/ Conde de Aranda, 20, 5D

ES-28001 Madrid, Spain

Medical and Scientific Director

M.D. Anderson España Cancer Center

C/ Gómez Hemans, 2

ES-28033 Madrid, Spain

email: rcolomer@seom.org; rcolomer@mdanderson.es 